\title{
Simulations of starting gas jets at low Mach numbers
}

\author{
Immaculada Iglesias, Marcos Vera, and Antonio L. Sánchez \\ Area de Mecánica de Fluidos, Universidad Carlos III de Madrid, 28911 Leganés, Spain \\ Amable Liñán \\ ETSI Aeronáuticos, Pl. Cardenal Cisneros 3, 28040 Madrid, Spain
}

(Received 10 November 2004; accepted 7 December 2004; published online 8 February 2005)

\begin{abstract}
The starting jet produced by the impulsively started discharge of a submerged gas stream of constant velocity through a circular orifice in a plane wall is investigated by integrating numerically the axisymmetric Navier-Stokes equations for moderately large values of the jet Reynolds number. The analysis is restricted to low-Mach-number jets, for which the jet-to-ambient temperature ratio $\gamma$ $=T_{j} / T_{o}$ emerges as the most relevant parameter. It is seen that the leading vortex approaches a quasisteady structure propagating at an almost constant velocity, which is larger for smaller values of $\gamma$. The action of the baroclinic torque in regions of nonuniform temperature leads to significant vorticity production, with a constant overall rate equal to that of an inviscid starting jet. (C) 2005 American Institute of Physics. [DOI: 10.1063/1.1858533]
\end{abstract}

Starting jets are produced by the discharge of fluid through a nozzle or orifice into an initially quiescent atmosphere, as can be done experimentally with a piston-cylinder mechanism by displacing a column of water through a circular nozzle into a larger water tank. The resulting unsteady jet flow shows a characteristic leading vortex ring, ${ }^{1}$ formed by the roll up of the vortex sheet that separates at the edge of the orifice, followed by a jet stem, whose annular vortex sheet evolves to form secondary vortices when the jet Reynolds number is sufficiently large.

Most previous works have considered the constant density case. The early stages of the process, including the roll up of the vortex sheet to form the vortex ring, have been studied both experimentally ${ }^{2}$ and numerically. ${ }^{3,4}$ Much less attention has been given to the long time evolution of starting jets as the leading vortex travels away while the jet stream continues dischaging from the orifice. Experiments performed with a piston-cylinder mechanism ${ }^{5}$ demonstrated, in particular, that the leading vortex stops entraining vorticity at a given time, pinching off from the jet stem, a phenomenon that was also observed in numerical simulations. ${ }^{6}$

The present Brief Communication investigates numerically starting jets formed by the impulsively started discharge of a gas stream of velocity $U_{j}$ from an orifice of diameter $D_{j}$ into a stagnant atmosphere of the same gas, a configuration sketched in Fig. 1. Jet temperatures different from the ambient value are considered in the analysis, which is restricted to values of the jet Mach number sufficiently small for the pressure variations to be negligible compared with the ambient pressure. To describe the resulting flow, the near-isobaric Navier-Stokes equations, written in axisymmetric form, are integrated numerically, as done for constant density in previous investigations. ${ }^{4,6,7}$ As in Ref. 6, the time range of integration is extended to values much larger than $D_{j} / U_{j}$, so that the long time behavior of the starting jet is appropriately described.

The application that motivates our interest in the problem is the ignition of a combustible mixture by a hot jet of reaction products. Despite the large Reynolds numbers typically encountered in realistic cases, the leading vortex that forms at the head of the transient jet provides a means for effective mixing at the molecular level, ${ }^{7}$ thereby leading to the formation of an ignition kernel. The combustion mode initiated by the hot jet depends critically on the value of $D_{j}$. Thus, for a given reactant mixture, there exists a critical value of $D_{j}$, of the order of the characteristic flame thickness $\left(\sim 10^{-4} \mathrm{~m}\right)$, below which ignition is precluded, while for larger values a deflagration forms. The initiation of a detonation requires much larger values of $D_{j}$, on the order of the detonation cell size $\left(\sim 10^{-1} \mathrm{~m}\right){ }^{8}$

To write the problem in nondimensional form, the values of $D_{j}$ and $U_{j}$ are used to scale the coordinates $x=x^{\prime} / D_{j}$ and $r=r^{\prime} / D_{j}$, the velocity components $v_{r}=v_{r}^{\prime} / U_{j}$ and $v_{x}=v_{x}^{\prime} / U_{j}$, the time $t=t^{\prime} /\left(D_{j} / U_{j}\right)$, and the pressure differences from the ambient value $p=\left(p^{\prime}-p_{a}^{\prime}\right) /\left(\rho_{j} U_{j}^{2}\right)$. Similarly, the inlet jet properties are used to define a dimensionless temperature, $T=T^{\prime} / T_{j}$, density, $\rho=\rho^{\prime} / \rho_{j}$, and viscosity, $\mu=\mu^{\prime} / \mu_{j}$. The axisymmetric continuity, momentum, and energy conservation equations are supplemented with the equation of state written in the near isobaric approximation, $\rho T=1$, and with the presumed power-law dependence $\mu=T^{\sigma}$ for the temperature variation of the viscosity, where the exponent is taken to be $\sigma=0.5$ in the computations. We choose to write the energy equation for the normalized temperature $\phi=\left(T^{\prime}-T_{o}\right) /\left(T_{j}\right.$ $-T_{o}$ ), defined to be unity in the jet and zero in the stagnant fluid. In the isothermal case, when $T=1$ everywhere in the flow field, this alternative variable constitutes a useful passive scalar to characterize the mixing process. Pressure variations and viscous dissipation are discarded in the energy equation, as corresponds to the low Mach-number jets considered here. Furthermore, a constant specific heat at constant pressure is assumed, along with a constant Prandtl number $\operatorname{Pr}=0.71$. As an additional simplification, buoyancy forces are neglected when writing the momentum equation, an appropriate approximation when the characteristic Froude 


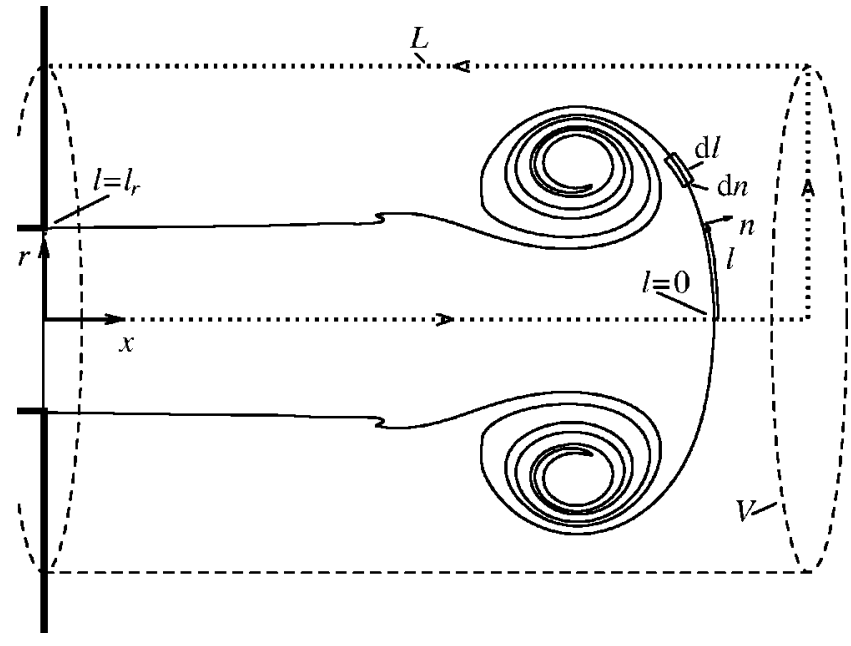

FIG. 1. A schematic representation of the starting jet, including the contour $L$ used to evaluate the total circulation $\Gamma$, the control volume $V$ used to integrate (3), and the coordinates $(l, r)$ used in evaluating (6).

number $U_{j}^{2} /\left(g D_{j}\right)$ is sufficiently large. Besides the Reynolds number, $\operatorname{Re}_{j}=\rho_{j} U_{j} D_{j} / \mu_{j}$, the solution is seen to depend only on the jet-to-ambient temperature ratio $\gamma=T_{j} / T_{o}$.

The conservation equations were integrated for $x>0$ and $t>0$ with boundary conditions at $x=0$ corresponding to a uniform jet, i.e., $v_{x}-1=v_{r}=\phi-1=0$ for $r<1 / 2$, and a nonslip adiabatic wall, i.e., $v_{r}=v_{x}=\partial \phi / \partial x=0$ for $r>1 / 2$. For $x$ $>0$, we impose the regularity conditions $\partial v_{x} / \partial r=v_{r}$ $=\partial \phi / \partial r=0$ along the axis $r=0$, together with an outflow condition for $x^{2}+r^{2} \rightarrow \infty$.

We use a prescribed constant uniform velocity profile at the jet exit $v_{x}=1$, a boundary condition also employed in previous calculations. ${ }^{6}$ Clearly, if our calculations were intended to reproduce a particular piston-cylinder experiment, then it would be necessary to describe the flow in the cylinder up to the piston, as done in Ref. 4 to reproduce the experiments of Ref. 2. The purpose here is not to reproduce a specific experiment, but rather to provide general understanding of starting gas jets, so that a simple boundary condition is preferred. Note that a uniform velocity profile could be obtained in the piston-cylinder experiment by incorporating a porous plate across the cylinder just upstream from the jet exit.

In the near-isobaric limit considered here, the outer stagnant fluid is set in motion at the initial instant to adjust to the boundary condition for the velocity imposed at the orifice. The resulting initial condition for the velocity corresponds to the potential flow generated by a uniform distribution of volumetric sources located at the orifice,

$$
\left(v_{x}, v_{r}\right)=\frac{1}{2 \pi} \int_{0}^{1 / 2} \int_{0}^{2 \pi} \frac{(x, r-\tilde{r} \cos \theta) \tilde{r} d \theta d \tilde{r}}{\left[x^{2}+(r-\tilde{r} \cos \theta)^{2}+(\widetilde{r} \sin \theta)^{2}\right]^{3 / 2}},
$$

while the initial temperature distribution is simply given by $\phi=0$. Note that the resulting nonzero radial velocity at $x=0$ results in a vorticity sheet that extends initially both across the orifice and on the wall.
The conservation equations were discretized following a cell-centered finite-volume procedure. A second-order upwind scheme was used to evaluate the face values of the variables for the convective terms, while second-order central differences were used for the diffusive terms. The pressure-velocity coupling was achieved with an algorithm of the SIMPLE family. The time discretization was implicit and first-order accurate. The computational domain $(0 \leqslant x$ $\leqslant 20,0 \leqslant r \leqslant 10$ ) was discretized in a block-structured grid. The initial grid consisted of a square grid with $\Delta x=\Delta r=0.5$ covering the whole domain plus a very refined grid in a region of dimensions $(0 \leqslant x \leqslant 1.5,0 \leqslant r \leqslant 1.25)$ around the jet exit, with minimum spacing $\Delta x=\Delta r=7.8125 \times 10^{-3}$. This original grid was dynamically adapted every ten time steps to extend the refined grid up to the region where the gradient of $\phi$ reached a threshold value of 0.001 . The number of computational cells ranged from 32042 for the original grid to more than 500000 for the more developed jets. The computational time step was dynamically determined based on the estimation of the truncation error associated with the time integration scheme, resulting in time steps of the order of $\Delta t \sim 10^{-3}$.

Figure 2 shows instantaneous maps of vorticity $\omega$ $=\partial v_{r} / \partial x-\partial v_{x} / \partial r$ and temperature $\phi$ obtained for $\gamma$ $=(1 / 3,1,3)$. To facilitate the comparison, different instants of time have been selected in the plot to make the penetration length approximately equal to 5 and a half diameters in all three cases. The Reynolds number based on the properties of the incoming fluid is taken to be $\operatorname{Re}_{j}=2000$. Since the kinematic viscosity of the ambient fluid is a factor $\gamma^{1+\sigma}$ smaller than that of the jet, increasing the value of $\gamma$ causes an increase in the effective Reynolds number of the flow, which has a noticeable effect on the instability of the trailing vortex sheet. Thus, no instability is observed in the jet stem for $\gamma$ $=1 / 3$ while at the same distance from the orifice a number of annular vortices have already developed for $\gamma=3$. The case $\gamma=1$ is intermediate, and shows already regions of concentrated vorticity which eventually develop into vortices for larger times. Vortex pairing enters to enrich the dynamics in the case $\gamma=3$, e.g., the leading vortex in the selected snapshot has just engulfed the vortex located immediately behind.

As can be seen, the leading vortex shows a fairly concentrated vorticity ring embedded in an annular region of uniform temperature, which results from the initial roll up of the vortex sheet. The plots corresponding to $\gamma=1 / 3$ and $\gamma$ $=3$ indicate that additional vorticity is produced by the baroclinic torque acting on regions of nonuniform density. For $\gamma=1 / 3$ the effect is less noticeable due to the increased thermal diffusivity, which tends to reduce the temperature gradients, so that appreciable vorticity, of the same sign as that being shed at the orifice rim, is only produced in the mixing layer surrounding the leading vortex. Larger temperature gradients, and therefore higher production rates, are seen for $\gamma$ $=3$, when production in the outer mixing layer surrounding the vortices yields negative vorticity. Note also the existence of vorticity of opposite sign on the sides of the stretched layer of cold gas trapped between the leading vortex core and the axis. The vorticity distribution of the leading vortex, initially connected to that of the jet stem, eventually discon- 


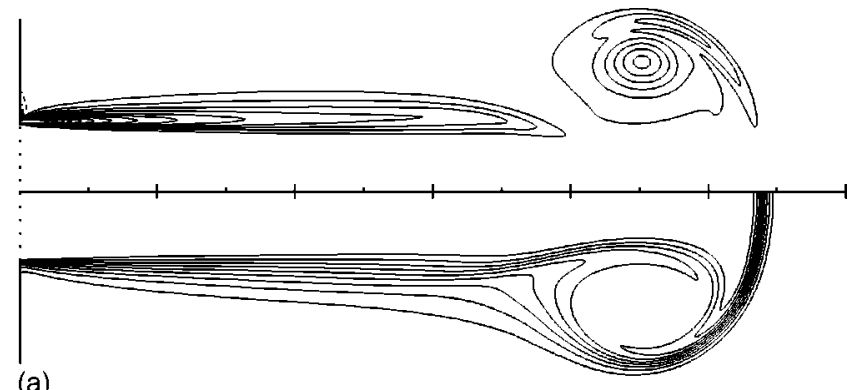

(a)

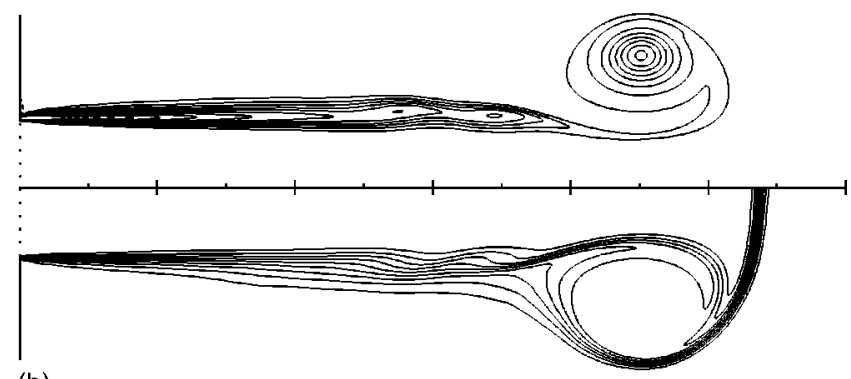

(b)

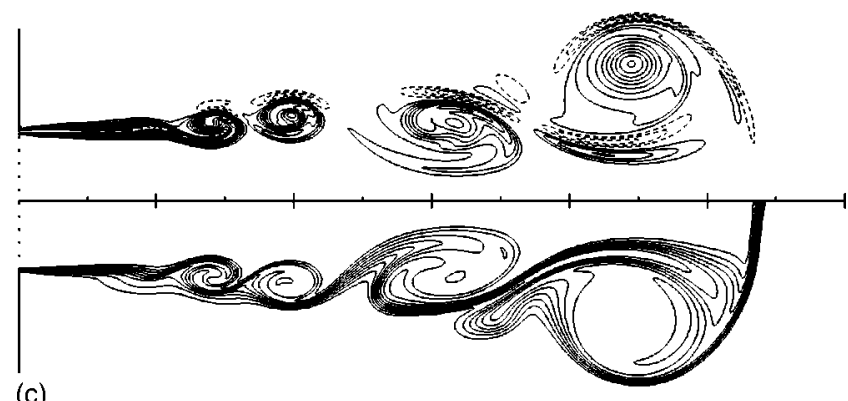

(c)

FIG. 2. Contours of $\omega$ (upper half) and $\phi$ (lower half) obtained for $\operatorname{Re}_{j}$ $=2000$ and (a) $\gamma=1 / 3$ at $t=8.44$, (b) $\gamma=1$ at $t=10.61$, and (c) $\gamma=3$ at $t$ $=14.30$; the isocontours of negative vorticity (dashed lines) range from $\omega$ $=-4$ to $\omega=-1$ in increments $\delta \omega=1$, whereas the isocontours of positive vorticity correspond to values of $\omega$ from $\omega=1$ to $\omega=16$ in increments $\delta \omega$ $=1$ and the temperature isocontours correspond to $\phi=0.1$ to $\phi=0.9$ in increments $\delta \phi=0.1$.

nects as the leading vortex pinches off, a phenomenon described in earlier work. ${ }^{5,6}$

Quantitative information regarding the leading vortex is presented in Fig. 3. In particular, the plot shows the evolution with time of the jet front, $x_{f}$, defined at each instant as the location along the axis where $\phi=0.5$. As can be seen, the front location, independent of $\mathrm{Re}_{j}$, depends strongly on $\gamma$. The associated vortex velocity, $U_{v}=d x_{f} / d t$, remains almost constant for $x_{f} \gtrsim 3$, so that the expected deceleration associated with viscous effects is almost imperceptible in the time range computed. The computation of $U_{v}$ by a least-square fit of the numerical results corresponding to $x_{f}>3$ gives $U_{v}$ $=(0.676,0.629,0.510,0.395,0.326)$ for $\operatorname{Re}_{j}=2000$ and $\gamma$ $=(1 / 5,1 / 3,1,3,5)$, while $U_{v}=(0.482,0.499)$ for $\gamma=1$ and $\operatorname{Re}_{j}=(500,1000)$.

To quantify the amount of vorticity present in the flow field, it is useful to introduce the total circulation $\Gamma$ $=\int_{L} \bar{v} \cdot d \bar{x}=\int_{S} \omega d r d x$, where the contour of integration $L$ and the associated rectangular surface $S$ are defined in Fig. 1. With the boundary condition used here at $x=0$, the circula-
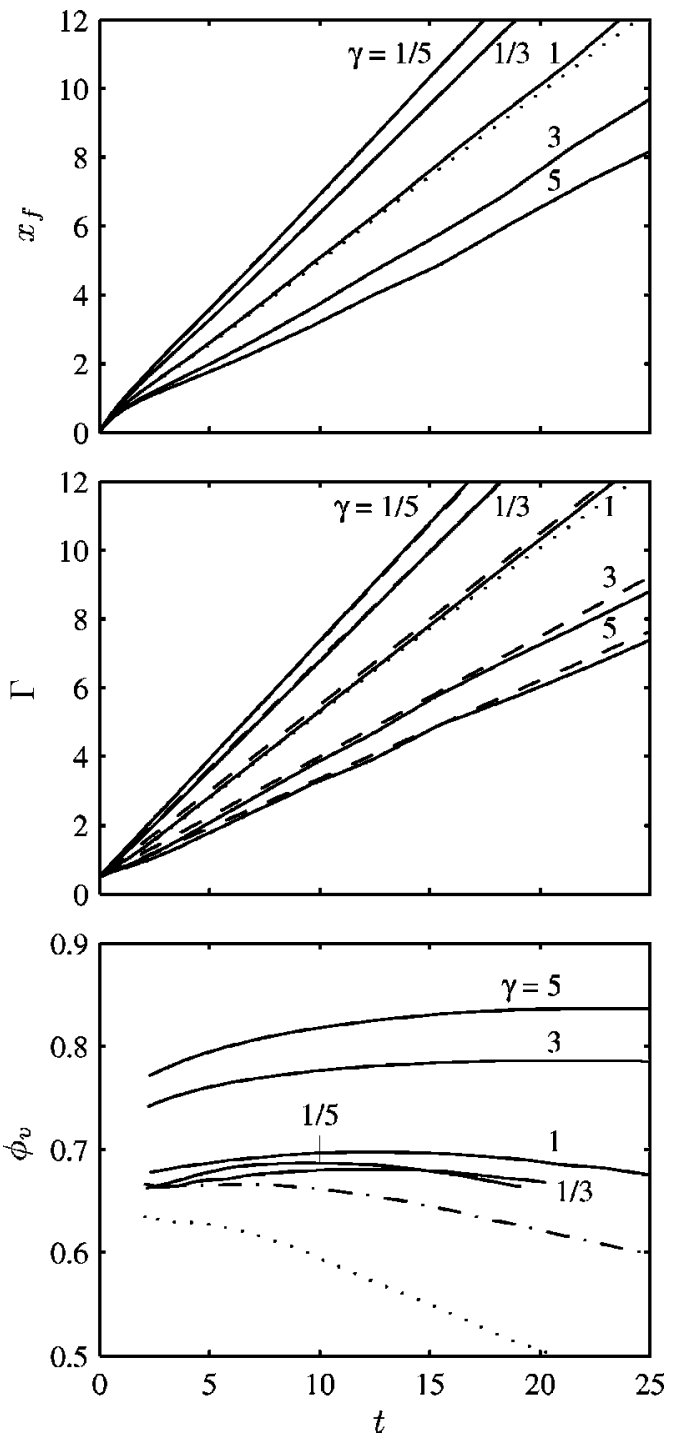

FIG. 3. Evolution with time of $x_{f}, \Gamma$, and $\phi_{v}$ for different values of $\gamma$ and $\mathrm{Re}_{j}$. Solid lines, $\mathrm{Re}_{j}=2000$; dotted lines, $\operatorname{Re}_{j}=500, \gamma=1$; dashed lines, prediction given by Eq. (2). The evolution of $\phi_{v}$ for $\operatorname{Re}_{j}=1000$ and $\gamma=1$ is given as a dash-dotted line, while the corresponding curves for $x_{f}$ and $\Gamma$, not shown in the figure, are virtually indistinguishable from those obtained for $\operatorname{Re}_{j}=2000$ and $\gamma=1$.

tion reduces to the line integral along the axis $\Gamma=\int_{0}^{\infty} v_{x} d x$ when $L$ is taken infinitely large. The numerical results were used to determine the evolution with time of $\Gamma$ for different values of $\gamma$ and $\mathrm{Re}_{j}$, giving the curves shown in Fig. 3. As can be seen, the circulation increases from a constant initial value $\Gamma=1 / 2$, independent of $\gamma$ and $\operatorname{Re}_{j}$, at an almost constant rate, which is smaller for increasing values of $\gamma$. The existence of a nonzero circulation at $t=0$, which is also observed in previous computations (see Figs. 3 and 4 of Ref. $6)$, is associated with the vorticity created during the impulsive acceleration of the flow. Thus, using (1) to evaluate the velocity $v_{x}=1-x /\left(x^{2}+1 / 4\right)^{1 / 2}$ along the axis, yields the initial circulation $\Gamma=\int_{0}^{\infty} v_{x} d_{x}=1 / 2$ observed in the computations.

The constant growth rate of the circulation seen in Fig. 3 is partly due to the vorticity being shed at the orifice rim and partly due to the baroclinic production. As can be seen, the 
resulting $\Gamma(t)$ compares extremely well with the value

$$
\Gamma=\frac{1}{2}+\frac{t}{2}\left[1+U_{v}^{2}(1-\gamma)\right]
$$

corresponding to an inviscid starting jet, with $U_{v}$ being evaluated in the comparisons from the numerical results. The above expression follows from integrating the vorticity equation for an axysimmetric flow,

$$
\frac{\partial}{\partial t^{\prime}}\left(\frac{\omega^{\prime}}{r^{\prime}}\right)+\nabla \cdot\left(\frac{\omega^{\prime}}{r^{\prime}} \bar{v}^{\prime}\right)=\frac{\left(\nabla \rho^{\prime} \wedge \nabla p^{\prime}\right)_{\theta}}{r^{\prime} \rho^{\prime 2}},
$$

in the cylindrical volume $V$ indicated in the sketch of Fig. 1, and dividing for convenience the result by $2 \pi$. Since $d V$ $=2 \pi r^{\prime} d r^{\prime} d x^{\prime}$, the first term in (3) yields

$$
\frac{1}{2 \pi} \frac{d}{d t^{\prime}} \int_{V} \frac{\omega^{\prime}}{r^{\prime}} d V=\frac{d}{d t^{\prime}} \int_{S} \omega^{\prime} d r^{\prime} d x^{\prime}=\frac{d \Gamma^{\prime}}{d t^{\prime}},
$$

whereas the second term yields the flux of $\omega^{\prime} / r^{\prime}$ across the surface $\Sigma$ limiting the cylindrical control volume $V$. Far from the jet, the motion of the initially stagnant fluid is irrotational, so that the flux of vorticity occurs only at the orifice, where $\omega^{\prime}=-\partial v_{x}^{\prime} / \partial r^{\prime}$ and one can write

$$
\frac{1}{2 \pi} \int_{\Sigma}\left(\frac{\omega^{\prime}}{r^{\prime}} \bar{v}^{\prime} \cdot \bar{n}\right) d \sigma=\int_{0}^{D_{j} / 2} v_{x}^{\prime} \frac{\partial v_{x}^{\prime}}{\partial r^{\prime}} d r^{\prime}=-\frac{U_{j}^{2}}{2} .
$$

For an inviscid flow, the density gradient is zero everywhere, except at the fluid surface separating the jet from the outer fluid, where it takes an infinite value. Therefore, to determine the contribution of the baroclinic torque, we use as coordinates the distance $l$ from the jet leading edge measured along the separating fluid surface and the accompanying local transverse coordinate $n$. In terms of these coordinates one obtains $\left(\boldsymbol{\nabla} \rho^{\prime} \wedge \nabla p^{\prime}\right)_{\theta}=\left(\partial \rho^{\prime} / \partial n\right)\left(\partial p^{\prime} / \partial l\right)$, which can be used to yield

$$
\int_{0-}^{0+} \frac{1}{\rho^{\prime 2}} \frac{\partial \rho^{\prime}}{\partial n} d n \int_{0}^{l_{r}} \frac{\partial p^{\prime}}{\partial l} d l=\left(p_{f}^{\prime}-p_{r}^{\prime}\right)\left(\frac{1}{\rho_{o}}-\frac{1}{\rho_{j}}\right),
$$

where $p_{f}^{\prime}$ is the pressure at the jet front $l=0$ and $p_{r}^{\prime}$ is the pressure at the orifice $\operatorname{rim} l=l_{r}$. This value rapidly approaches the ambient pressure $p_{a}^{\prime}$ as the leading vortex moves away from the orifice rim, so that finally

$$
\frac{d \Gamma^{\prime}}{d t^{\prime}}=\frac{U_{j}^{2}}{2}+\left(p_{f}^{\prime}-p_{a}^{\prime}\right)\left(\frac{1}{\rho_{o}}-\frac{1}{\rho_{j}}\right) .
$$

Since the velocity of the leading vortex $U_{v}^{\prime}$ is almost constant, in a reference frame moving with the vortex conservation of total head along the axis gives $p_{f}^{\prime}-p_{a}^{\prime}=\rho_{o} U_{v}^{\prime 2} / 2$. In- tegrating (7) with this constant overpressure and scaling the result with $U_{j} D_{j}$ finally yields (2).

Figure 3 also shows the time evolution of $\phi_{v}$, the value of the temperature at the vortex center, defined as the location where $\omega$ reaches its peak value. The variations of the initial value of $\phi_{v}$ for different $\operatorname{Re}_{j}$ and $\gamma$ are not extremely large, i.e., the resulting $\phi_{v}$ lies typically in the range 0.65 $\lesssim \phi_{v} \lesssim 0.75$, indicating that during the early stages of vortex formation the vortex entrains mainly jet fluid. The subsequent evolution suggests that the leading vortex continues entraining fluid from the near axis region, where the temperature remains equal to that of the jet during a considerable period of time, causing an initial increase of $\phi_{v}$. This effect is partly balanced by conduction heat losses towards the ambient gas, which eventually dominate, causing the temperature to slowly decrease for sufficiently large times. The latter effect is less important for larger $\operatorname{Re}_{j}$ and also for larger $\gamma$, when the thermal diffusivity of the relatively cold outer fluid is smaller than that of the jet, thereby reducing the rate of heat conduction towards the ambient.

The results presented motivate further research on nonisothermal gaseous starting jets. It can be expected that a study of the kind performed by Pullin ${ }^{9}$ for the inviscid kinematics of the roll up process would provide additional information on the initial value of $\phi_{v}$, of interest in ignition applications. In addition, the dynamics of steady vortex rings of variable density should be investigated to clarify the dependence of $U_{v}$ on $\gamma$.

This collaborative research was supported by the Spanish MCyT under Project No. DPI2002-04550-C07.

${ }^{1}$ K. Shariff and A. Leonard, "Vortex rings," Annu. Rev. Fluid Mech. 24, 235 (1992).

${ }^{2} \mathrm{~N}$. Didden, "On the formation of vortex rings: rolling-up and production of circulation," Z. Angew. Math. Phys. 30, 101 (1979).

${ }^{3} \mathrm{M}$. Nitsche and R. Krasny, "A numerical study of vortex ring formation at the edge of a circular tube," J. Fluid Mech. 276, 139 (1994).

${ }^{4}$ R. S. Heeg and N. Riley, "Simulations of the formation of an axisymmetric vortex ring," J. Fluid Mech. 339, 199 (1997).

${ }^{5}$ M. Gharib, E. Rambod, and K. Shariff, "A universal time scale for vortex ring formation," J. Fluid Mech. 360, 121 (1998).

${ }^{6}$ M. Rosenfeld, E. Rambod, and M. Gharib, "Circulation and formation number of laminar vortex rings," J. Fluid Mech. 376, 297 (1998).

${ }^{7}$ R. Verzicco and P. Orlandi, "Mixedness in the formation of a vortex ring," Phys. Fluids 7, 1513 (1995).

${ }^{8}$ R. Knystautas, J. H. S. Lee, I. Moen, and H. G. Wagner, "Direct initiation of a spherical detonation by a hot turbulent gas jet," Proc. Combust. Inst. 17, 1235 (1979).

${ }^{9}$ D. I. Pullin, "Vortex ring formation at tube and orifice openings," Phys. Fluids 22, 401 (1979). 
Physics of Fluids is copyrighted by the American Institute of Physics (AIP). Redistribution of journal material is subject to the AIP online journal license and/or AIP copyright. For more information, see http://ojps.aip.org/phf/phfcr.jsp 